\title{
The "no-touch" harvesting technique for vein grafts in coronary artery bypass surgery preserves an intact vasa vasorum
}

\author{
Mats Dreifaldt, ${ }^{\mathrm{a}}$ Domingos S. R. Souza, ${ }^{\mathrm{a}}$ Andrzej Loesch, ${ }^{\mathrm{b}}$ John R. Muddle, ${ }^{\mathrm{c}}$ Mats G. Karlsson, ${ }^{\mathrm{d}}$ \\ Derek Filbey, ${ }^{\mathrm{d}}$ Lennart Bodin, ${ }^{\mathrm{e}}$ Lars Norgren, ${ }^{\mathrm{f}}$ and Michael R. Dashwood ${ }^{\mathrm{g}}$
}

Objectives: Our objective was to evaluate the impact of vein graft harvesting technique on structure and function of vasa vasorum.

\begin{abstract}
Methods: Paired segments of great saphenous veins harvested either with conventional harvesting technique or no-touch technique were obtained from 9 consecutive patients undergoing coronary artery bypass grafting. Quantitative measurements, using immunohistochemistry and morphometry, were performed. Ultrastructural analyses of vasa vasorum were performed with electron microscopy. Video footage of superficial vasa vasorum in an implanted saphenous vein graft harvested with the no-touch technique was captured during a coronary bypass operation and is presented for online viewing.
\end{abstract}

Results: The total area of vasa vasorum in vein grafts harvested with the conventional technique was significantly reduced both in the media $(P=.007)$ and in the adventitia $(P=.014)$ compared with vein grafts harvested with the no-touch technique. Ultrastructural findings indicated that the no-touch technique preserved an intact vasa vasorum whereas the conventional technique did not. Video footage showed retrograde flow in the vasa vasorum in vein graft harvested with the no-touch technique.

Conclusions: These findings show that the no-touch technique for saphenous vein graft harvesting for coronary bypass grafting preserves an intact vasa vasorum. This could represent one of the mechanisms underlying the improved patency of saphenous vein grafts harvested with this technique. (J Thorac Cardiovasc Surg 2011;141:145-50)

Video clip is available online.

The use of the saphenous vein (SV) as a conduit for coronary artery bypass grafting (CABG) was first described by Favaloro $^{1} 40$ years ago, and this vein remains the most widely used graft despite a patency rate of about $60 \%$ at 10 years compared with more than $90 \%$ for the internal thoracic artery. ${ }^{2,3}$

With conventional harvesting technique (CT), the SV is stripped of its surrounding tissue and distended to overcome spasm, procedures that are known to cause vein wall damage. ${ }^{4}$ In the mid-1990s, a novel vein graft harvesting tech-

\footnotetext{
From the Departments of Thoracic and Cardiovascular Surgery, ${ }^{a}$ Laboratory Medicine,${ }^{\mathrm{d}}$ Biostatistics, ${ }^{\mathrm{e}}$ and Surgery, ${ }^{\mathrm{f}}$ Örebro University Hospital, Örebro, Sweden; and the Departments of Inflammation, ${ }^{b}$ Neurological Sciences, ${ }^{c}$ and Clinical Biochemistry, ${ }^{\mathrm{g}}$ UCL Medical School, Royal Free Campus, London, United Kingdom. The study was granted by the local Research Committee, Örebro University Hospital and the local Society of Medicine in Örebro.

Supported in part by the Wellcome Trust (to A.L.).

Disclosures: Authors have nothing to disclose with regard to commercial support.

Received for publication March 31, 2009; revisions received Dec 15, 2009; accepted for publication Feb 1, 2010; available ahead of print April 12, 2010.

Address for reprints: Dr Mats Dreifaldt, Department of Thoracic and Cardiovascular

Surgery, Örebro University Hospital, SE-701 85 Örebro, Sweden (E-mail: mats. dreifaldt@orebroll.se).

0022-5223/\$36.00

Copyright $($ C 2011 by The American Association for Thoracic Surgery

doi:10.1016/j.jtcvs.2010.02.005
}

nique was introduced, the no-touch technique (NT), in which the SV is harvested with its pedicle of surrounding tissue. ${ }^{5}$ This technique prevents the graft from spasm, and distention is therefore not needed. ${ }^{6}$ A prospective randomized trial showed a significantly increased patency rate at 8.5 years for SVs harvested with NT compared with CT $(90 \%$ vs $76 \%)$.

The pedicle of tissue surrounding the SV is composed of fat, elastic tissue, nerves, and vasa vasorum, a network of nourishing microvessels supplying nutrients and oxygen to the blood vessel wall. Vasa vasorum is more pronounced in muscular veins than in arteries and extends deep into the tunica media. With CT the surrounding tissue, including parts of the adventitia, is stripped off and the vasa vasorum is damaged. The aim of this study was to evaluate the morphology and function of vasa vasorum in SV grafts harvested with NT compared with CT. Quantitative morphometric analyses of vasa vasorum within the vein graft wall were performed using immunohistochemistry and an automatic imaging system. Ultrastructural changes were studied using transmission (TEM) and scanning (SEM) electron microscopy. The restoration of blood flow through the vasa vasorum was recorded by video camera during a typical/ representative $\mathrm{CABG}$ procedure in which vein grafts harvested with NT were used.

\section{PATIENTS AND METHODS}

After ethics committee approval and patients' written consent, an additional 7-cm SV segment was obtained from 9 consecutive patients who 


$$
\begin{aligned}
& \text { Abbreviations and Acronyms } \\
& \text { CABG }=\text { coronary artery bypass grafting } \\
& \text { CT }=\text { conventional harvesting technique } \\
& \text { NT }=\text { no-touch technique } \\
& \text { SD }=\text { standard deviation } \\
& \text { SEM }=\text { scanning electron microscopy } \\
& \text { SV }=\text { saphenous vein } \\
& \text { TEM }=\text { transmission electron microscopy }
\end{aligned}
$$

underwent CABG at Örebro University Hospital (9/9 male, 51-65 years of age).

From each patient half of the additional SV segment was harvested with $\mathrm{CT}$, during which procedure the vein was stripped of surrounding tissue and distended with $0.9 \%$ saline solution at a controlled pressure of $300 \mathrm{~mm} \mathrm{Hg}$ for 1 minute. The other half of the SV segment was harvested with NT, in which the vein was neither stripped nor distended.

The samples were then immediately immersion-fixed for 5 hours at $4{ }^{\circ} \mathrm{C}$ in $4 \%$ formaldehyde for light microscopic immunohistochemistry and in $4 \%$ paraformaldehyde and $2 \%$ glutaraldehyde (in 0.1 -mol phosphate buffer, $\mathrm{pH}$ 7.4) for the SEM and TEM study.

\section{Immunohistochemistry}

Formaldehyde-fixed tissues were paraffin embedded and cut into $4-\mu \mathrm{m}$ transverse sections onto DAKO ChemMate Capillary gap microscope slides (DakoCytomation Denmark A/S, Gostrup, Denmark) and placed in an oven maintained at $60^{\circ} \mathrm{C}$ for 1 hour. The slides were then subjected to immunohistochemistry as follows. After initial deparaffinization, antigen was retrieved by microwaving the slides in a Tris-ethylenediaminetetraacetic acid buffer, $\mathrm{pH} 9$ at $650 \mathrm{~W}$, for 30 minutes. So that endothelial cells could be identified, immunohistochemistry was performed according to ChemMate DAKO EnVision Detection Kit (DakoCytomation Denmark), with primary antibody incubation for 25 minutes at room temperature (CD34, clone QBEnd-10, 1:100 DakoCytomation Denmark). Negative control slides were prepared by substituting primary antibody for Dako ChemMate antibody diluent (DakoCytomation Denmark). Images from slide-mounted sections were captured (via times 12.5 objective) with a Zeiss Axiocam MRc5 color camera (Carl Zeiss AG, Oberkochen, Germany) on an automated Zeiss Axioplan Microscope using Zeiss Axiovision software (final magnification of sections viewed on monitor $>300$ times). The images were normalized and the borders of media (between the internal and external elastic laminae) and adventitia (between external elastic laminae and the border of surrounding tissue) were manually defined. Measurements of media and adventitial area within the defined borders were performed. Endothelial cells and each area of detected vasa vasorum were automatically identified by the imaging system. Total vasa vasorum area (the sum of all areas detected) and density (number of detected vasa vasorum per square millimeter) were calculated within the media and the adventitia, respectively. In most cases, 2 sections were analyzed for each sample and the mean values recorded. Image processing and analysis was carried out using Zeiss KS400 software.

\section{TEM}

After fixation, the specimens were postfixed in $1 \%$ osmium tetroxide (in $0.1-\mathrm{mol} / \mathrm{L}$ sodium cacodylate buffer) for 1 hour at $4{ }^{\circ} \mathrm{C}$, washed in sodium cacodylate buffer, and then dehydrated by gradually increasing concentrations of ethanol, followed by propylene oxide. Specimens were embedded in Araldite adhesive (Huntsman Advanced Materials, Hong Kong) and polymerized at $60^{\circ} \mathrm{C}$ for 48 hours. Ultrathin sections $(80-85 \mathrm{~nm})$ were stained with uranyl acetate and lead citrate and subsequently examined with a Philips CM-120 TEM (Philips, Andover, Mass).
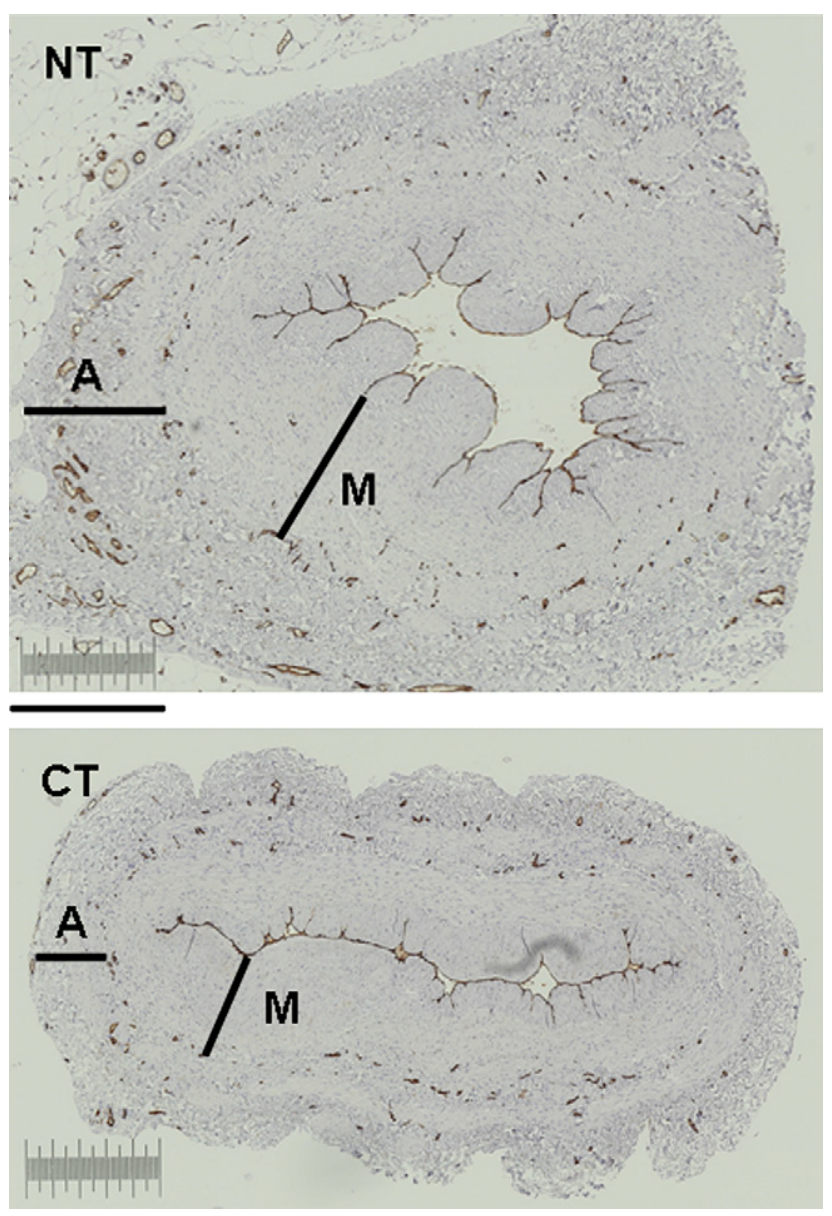

FIGURE 1. Representative transverse sections of no-touch (NT) and conventionally $(C T)$ harvested saphenous vein segments from a coronary bypass patient used for CD34 immunolabeling to identify endothelial cells (brown reaction product). A, Adventitia; $M$, media. Scale bar $=0.5 \mathrm{~mm}$.

\section{SEM}

After fixation, the specimens were washed in phosphate buffer and then sectioned either longitudinally or transversally. The specimens were then post-fixed in $1 \%$ osmium tetroxide (in $0.1-$ mol/L sodium cacodylate buffer)

TABLE 1. Vasa vasorum in SV grafts for NT and CT for 9 patients and 2 grafts per patient with 1 graft for each harvesting technique

\begin{tabular}{lccc}
\hline & NT & CT & Difference NT-CT \\
& Mean (SD) & Mean (SD) & $\boldsymbol{P}^{*}$ \\
\hline Media & & & \\
$\quad$ Density & $48.0(13.9)$ & $50.9(21.8)$ & .65 \\
Size & $138.3(21.1)$ & $129.7(23.1)$ & .18 \\
Total area & $27,502(13,946)$ & $18,359(9241)$ & .007 \\
& & & \\
Adventitia & & & .61 \\
$\quad$ Density & $94.5(24.7)$ & $101.9(48.8)$ & .11 \\
Size & $198.6(46.1)$ & $164.1(44.5)$ & .014 \\
$\quad$ Total area & $36,472(12,826)$ & $22,206(7598)$ & \\
\hline
\end{tabular}

$S V$, Saphenous vein; $N T$, no-touch technique; $C T$, conventional harvesting technique; $S D$, standard deviation. ${ }^{*} P$ values from testing equality in mean values using a mixed model analysis with allowance for 2 grafts from each patient. Density = numbers per square millimeter. Size and total area $=$ square micrometers. 

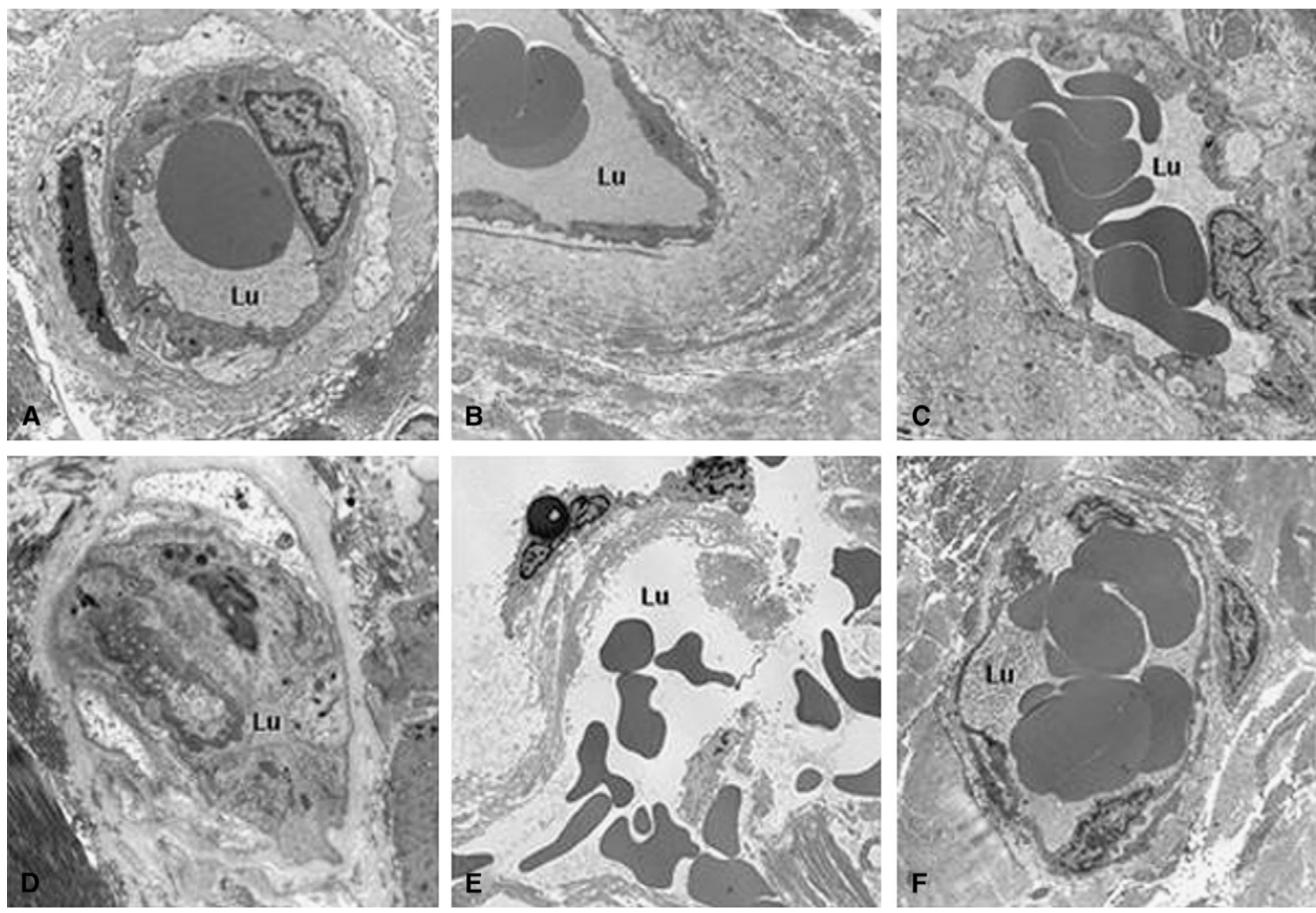

FIGURE 2. Transmission electron microscopic features of vasa vasorum in NT (A-C) and CT (D-E) saphenous vein graft preparations. Note the open lumen in all vasa vessels in NT preparations (A-C); also note the presence of red blood cells within the luminal space (lu). In CT preparations vasa are frequently collapsed or constricted so that the luminal space is closed and not readily visible (D). In (E) note the damage to a vasa vessel by CT harvesting; also note red blood cells outside the vascular pool. In contrast, image $(\mathrm{F})$ shows a rather well-preserved vessel. Original magnifications: A, D, $\times 5600$; B, E, F, $\times 2650$; C, $\times 4400$.

for 1 hour at $4^{\circ} \mathrm{C}$, washed in sodium cacodylate buffer, and dehydrated by gradually increasing concentrations of ethanol (from $25 \%$ to $100 \%$ ). Specimens were transferred to acetone (two exchanges of 10 minutes each) and dried in the critical-point dryer (replacement of acetone with liquid carbon dioxide at critical point) and then mounted on aluminium pin stubs $(32 \mathrm{~mm}$ in diameter) with sticky carbon tabs. They were subsequently surfacecoated with gold in a SC500 Sputter Coater Operator (Elexience, Paris, France) and examined with a JSM-5410LV SEM (JEOL Ltd, Tokyo, Japan). The images were captured electronically.

\section{Video Uptake}

During a CABG operation the flow through the superficial network of vasa vasorum in an SV graft harvested with NT was captured, when the graft was perfused via the arterial line from the heart-lung machine, after the distal anastomoses had been performed, using a digital video camera recorder (Panasonic DVX 100A; Panasonic Corporation, Seacaucus, NJ).

\section{Statistical Analysis}

The distributions of vasa microvessel density and area were described by mean and standard deviation (SD). Differences of density and area between the segments (samples) collected with the NT and the CT were analyzed in a mixed model analysis. ${ }^{8}$ This was done inasmuch as 2 segments were taken from each patient and the correlation between samples from the same patient had to be taken into account. The technical specification of the mixed model included density and area as continuous outcome variables, the harvesting technique as a binary factor (CT or NT) and a correlation structure with compound symmetry, and the degrees of freedom given by the Satterthwaite method. The raw data did not reject the normality assumptions, tested with the Shapiro-Wilk test, but as an extra precaution we also performed the analysis on logarithmically transformed values. Because this analysis gave similar results, we report results only for the untransformed data. Statistical analysis was performed using SAS software, version 9.1.3 (SAS Institute, Inc, Cary, NC).

\section{RESULTS}

\section{Immunohistochemistry}

Quantitative measurements showed that the area of the media in CT preparations was reduced by $29 \%$ (SD 16\%) compared with that of NT harvested veins $(\mathrm{CT}=2.97$ $\mathrm{mm}^{2}$ [SD $0.99 \mathrm{~mm}^{2}$ ] vs NT $=4.42 \mathrm{~mm}^{2}$ [SD $\left.2.18 \mathrm{~mm}^{2}\right]$ ). The area of the adventitia in CT preparations was reduced by $38 \%$ (SD $24 \%$ ) compared with the adventitial area of NT grafts $\left(C T=1.7 \mathrm{~mm}^{2}\left[\mathrm{SD} 0.7 \mathrm{~mm}^{2}\right]\right.$ vs NT $=2.4$ $\left.\mathrm{mm}^{2}\left[\mathrm{SD} 1.0 \mathrm{~mm}^{2}\right]\right)$. The differences did not reach statistical 


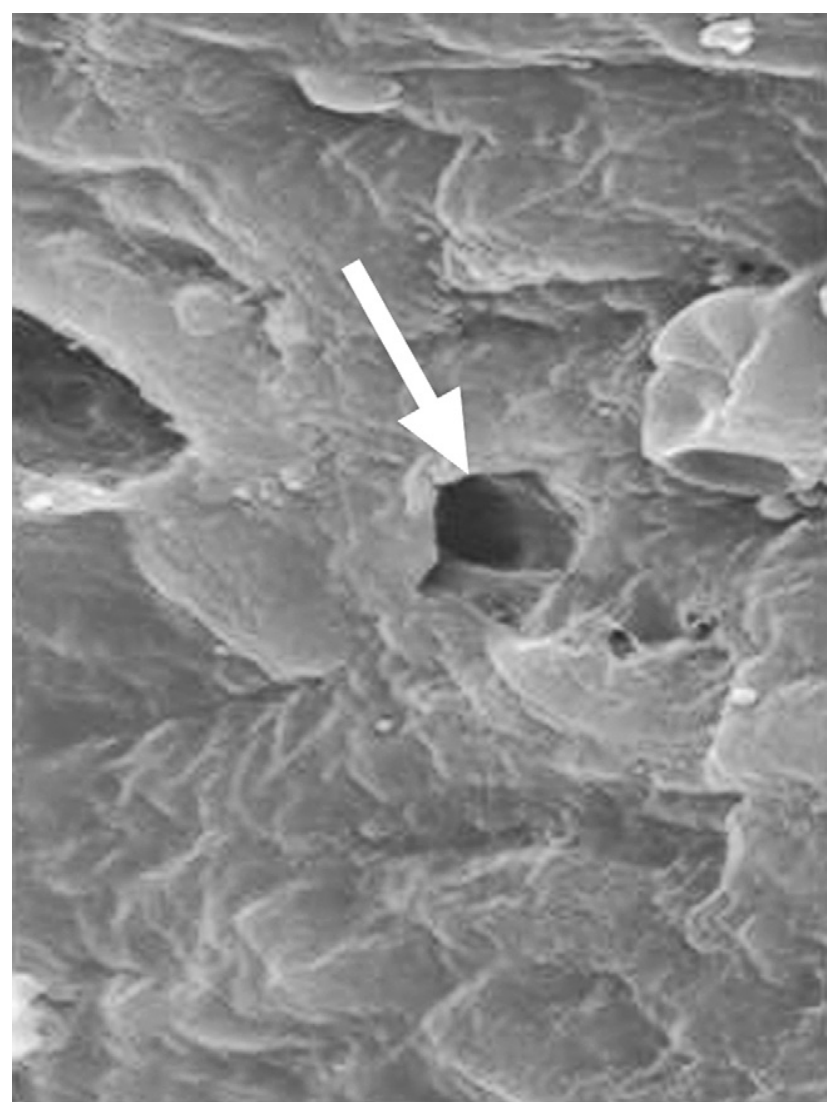

FIGURE 3. Scanning electron microscopy showing a potential opening of vasa vasorum (arrow) in the endothelium in an NT vein graft. Original magnification $\times 3000$.

significance (Figure 1). The mean ratio of the density between vasa vasorum in the media and the adventitia did not differ significantly between NT and CT preparations either $(P=.65, P=.61)$. In both preparations the mean density of the adventitial vasa vasorum was twice that of the media. The size of each detected microvessel of vasa vasorum showed a tendency to be smaller in $\mathrm{CT}$ vein grafts than in NT vein grafts, both in the media $(P=.18)$ and in the adventitia $(P=.11)$. Considering the density and size of vasa vasorum and the area of media and adventitia in CT and NT vein grafts, the total area of vasa vasorum in the media was significantly reduced in CT compared with NT vein grafts $(P=.007)$, as was the total area of vasa vasorum in the adventitia $(P=.014)($ Table 1$)$.

\section{TEM}

No quantitative assessment of electron microscopy was performed. TEM confirmed that in NT preparations a rich network of vasa vasorum was present, located in the adventitia, the adventitial/medial border. and the outer media. The lumen of these vessels was characteristically open (Figure 2, $A$ to $C$ ).

Within the remnant of the adventitia of CT preparations, both structurally changed and unchanged vessels were observed, but a network of vasa vasorum was difficult to find with TEM (Figure 2, $D$ to $F$ ). Vessels of smaller diameter were particularly affected, collapsed (Figure $2, D$ ) or occluded with red cells. Some of the vessels were severely damaged (Figure 2,E). Other vessels were relatively unaffected, with a normal structure and an open lumen. These vessels were usually arterioles or venules of larger diameter, which were still present in the remaining portion of the adventitia (Figure 2, F).

\section{SEM}

SEM showed structures indicating luminal openings in the endothelium of the SV only in those grafts that were harvested with NT (Figure 3).

\section{Clinical Observation}

A functional vasa vasorum was confirmed and recorded during one typical/representative CABG operation in which an SV graft harvested with NT showed a rapid retrograde filling of superficial vasa vasorum in the surrounding perivascular tissue. The NT vein graft was perfused with blood from the arterial line from the heart-lung machine before completion of the proximal anastomoses according to our routine procedure.

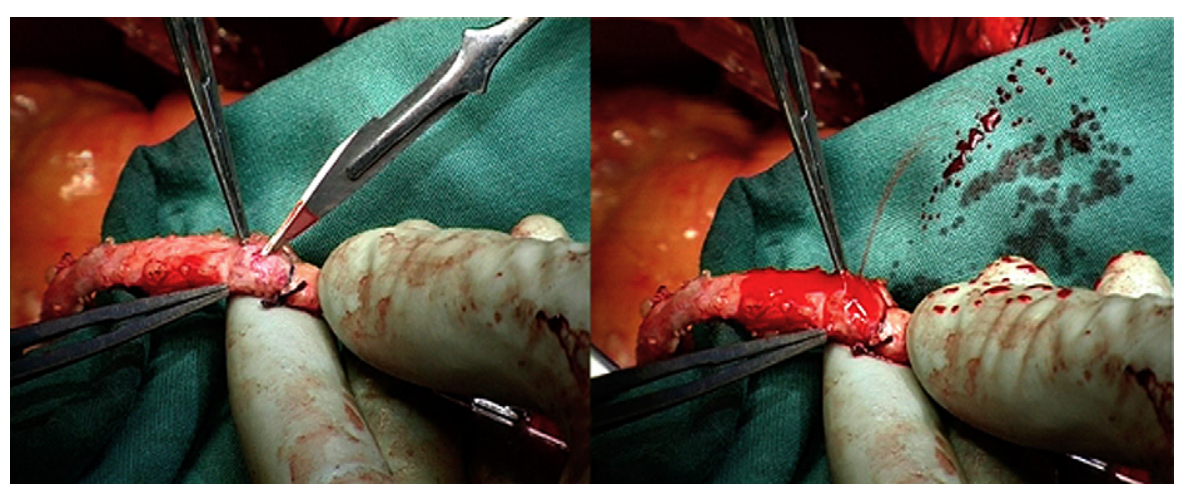

FIGURE 4. Bleeding from an incised vasa vasorum of SV graft harvested with the NT technique (screen shot from the video). 
Compression, using forceps, of vasa vasorum interrupted flow through these microvessels that refilled as the forceps were released. Furthermore, bleeding occurred when the vasa vasorum was cut with a scalpel, confirming the existence of a communication between vasa vasorum and the lumen of the vein (Figure 4).

These findings were video recorded and are presented for online viewing (see Video 1).

\section{DISCUSSION}

We have shown that the NT for SV graft harvesting preserves an intact vasa vasorum. When the SV is prepared using the CT, the adventitia is partially removed, a procedure that will result in damage to the vasa vasorum. There is experimental evidence that interruption of blood flow through the vasa vasorum in arteries by a close-fitting external cuff results in transmural ischemia that leads to neointimal hyperplasia and the eventual appearance of atherosclerotic lesions. ${ }^{9}$ Inasmuch as the requirement for a functional vasa vasorum in veins is greater than in arteries, where oxygen/ nutrients are obtained by diffusion from luminal blood, damage to this microvessel network is likely to have more serious consequences. Removal and disconnection of the vasa vasorum during conventional SV graft harvesting may therefore be expected to initiate similar events and could contribute to graft failure.

In large veins, such as the SV, there is an interaction between the vasa vasorum and the mechanical properties of the vessel wall. The CT for vein graft harvesting renders the vein graft spastic and therefore it has to be distended. Increased luminal pressure induces a shape change in the vasa vasorum, from their original circular appearance to an elliptical shape, and this results in a reduction of blood flow in the media of the vessel causing loss of the wall elasticity. ${ }^{10}$ In CT preparations the total vasa vasorum area was 37\% lower than that in NT preparations, reflecting a shape change and diminished area of the vasa vasorum in CT vein grafts. The vasa vasorum of the SV is densely innervated mainly by unmyelinated sympathetic nerves, and the presence of several layers of surrounding smooth muscle cells implies that the vessels of the vasa vasorum actively regulate their own tone. ${ }^{11}$ It is likely that surgical and distention-induced trauma causes constriction of vasa vasorum and consequent reduction in blood supply to the vessel wall.

In this study, as previously shown, ${ }^{12,13}$ there is TEM ultrastructural evidence that the vasa vasorum in vein grafts prepared by CT are disrupted and collapsed and exhibit erythrocyte "'plugging." These events would potentially occlude the vasa vasorum system, and in the present study we found such occlusions present even in the two CT samples that had an intact adventitia as demonstrated by TEM. SEM showed a possible communication between vasa vasorum and the lumen of the SV, which confirms our previous results. ${ }^{14}$
The suggestion made by Crotty ${ }^{15}$ that luminal blood flow to the vein graft wall may occur via the vasa vasorum is supported by our video footage, which clearly showed blood flow through these vessels in NT vein grafts after their implantation in CABG. We suggest that if the adventitial layer is left intact the vasa vasorum is preserved and maintains a "sealed system" that restores blood flow to the graft wall from the SV lumen.

The preservation of vasa vasorum in the vein graft has the potential to prevent ischemia and malnutrition, providing an additional mechanism explaining the improved long-term patency for SV grafts harvested with NT. ${ }^{16-18}$

\section{Limitation of the Study}

The quantitative measurements were carried out on veins obtained from a small number of patients. Inasmuch as CT and NT preparations were performed of the same veins from each patient, the comparison is relevant. Also, there was a relatively high variability in measurements that reflects the individual variations between patients, variation of vessel wall thickness within the same vein, and the inevitable variation associated with stripping the adventitia when preparing by the CT.

\section{CONCLUSIONS}

Our findings show that the NT for SV graft harvesting for CABG preserves an intact vasa vasorum. This could represent one of the mechanisms underlying the improved patency of SV grafts harvested with this technique.

We express our appreciation to Dr Tom Crotty, Department of Physiology, University College Cork, Cork, Ireland, and Dr Stephen E. Fremes, Division of Cardiac and Vascular Surgery, Sunnybrook Health Sciences Centre, Toronto, Ontario, Canada, for reviewing this article.

\section{References}

1. Favaloro RG. Saphenous vein graft in the surgical treatment of coronary artery disease: operative technique. J Thorac Cardiovasc Surg. 1969;58:178-85.

2. Dion R, Glineur D, Derouck D, Verhelst R, Noirhomme P, El Khoury G, et al. Complementary saphenous grafting: long-term follow-up. J Thorac Cardiovasc Surg. 2001;122:296-304

3. Lytle BW, Loop FD, Cosgrove DM, Ratliff NB, Easley K, Taylor PC. Long-term (5 to 12 years) serial studies of internal mammary artery and saphenous vein coronary bypass grafts. J Thorac Cardiovasc Surg. 1985;89:248-58.

4. Tsui JC, Souza DS, Filbey D, Bomfim V, Dashwood MR. Preserved endothelial integrity and nitric oxide synthase in saphenous vein grafts harvested by a "notouch"' technique. Br J Surg. 2001;88:1209-15.

5. Souza DA. new no-touch preparation technique. Technical notes. Scand J Thorac Cardiovasc Surg. 1996;30:41-4.

6. Tsui JCS, Dashwood MR. Recent strategies to reduce vein graft occlusion: a need to limit the effect of vascular damage. Eur J Vasc Endovasc Surg. 2002;23:202-8.

7. Souza DS, Johansson B, Bojo L, Karlsson R, Geijer H, Filbey D, et al. Harvesting the saphenous vein with surrounding tissue for CABG provides long-term graft patency comparable to the left internal thoracic artery: results of a randomized longitudinal trial. J Thorac Cardiovasc Surg. 2006;132:373-8.

8. Brown HPR. Applied mixed models in medicine. 2nd ed. Chichester(UK): John Wiley \& Sons; 2006. 
9. Barker SG, Talbert A, Cottam S, Baskerville PA, Martin JF. Arterial intimal hyperplasia after occlusion of the adventitial vasa vasorum in the pig. Arterioscler Thromb. 1993;13:70-7.

10. Maurice G, Wang X, Lehalle B, Stoltz JF. [Modeling of elastic deformation and vascular resistance of arterial and venous vasa vasorum]. J Mal Vasc. 1998;23:282-8.

11. Scotland RS, Vallance PJ, Ahluwalia A. Endogenous factors involved in regulation of tone of arterial vasa vasorum: implications for conduit vessel physiology. Cardiovasc Res. 2000;46:403-11.

12. Ahmed SR, Johansson BL, Karlsson MG, Souza DS, Dashwood MR, Loesch A. Human saphenous vein and coronary bypass surgery: ultrastructural aspects of conventional and "no-touch", vein graft preparations. Histol Histopathol. 2004; 19:421-33.

13. Vasilakis V, Dashwood MR, Souza DS, Loesch A. Human saphenous vein and coronary bypass surgery: scanning electron microscopy of conventional and “no-touch" vein grafts. Vasc Dis Prevent. 2004;1:133-9.
14. Souza DS, Christofferson RH, Bomfim V, Filbey D. No-touch“" technique using saphenous vein harvested with its surrounding tissue for coronary artery bypass grafting maintains an intact endothelium. Scand Cardiovasc J. 1999; 33:323-9.

15. Crotty TP. The venous valve agger and plasma noradrenaline-mediated venodilator feedback. Phlebology. 2007;22:116-30.

16. Kachlik D, Lametschwandtner A, Rejmontova J, Stingl J, Vanek I. Vasa vasorum of the human great saphenous vein. Surg Radiol Anat. 2003;24:377-81.

17. Dashwood MR, Anand R, Loesch A, Souza DS. Hypothesis: a potential role for the vasa vasorum in the maintenance of vein graft patency. Angiology. 2004;55: 385-95.

18. Dashwood MR, Savage K, Tsui JCS, Dooley A, Shaw SD, Fernandez-Alfonso M. Retaining perivascular tissue of human saphenous vein grafts protects against surgical and distension-induced damage and preserves eNOS and NOS activity. $J$ Thorac Cardiovasc Surg. 2009;138:334-40. 